\title{
Diabetes Mellitus and the Development of Lumbar Canal Stenosis: Is There Any Relevance?
}

\author{
Ghanshyam Kakadiya ${ }^{1}$, Kalpesh Saindane ${ }^{1}$, Yogesh Soni ${ }^{2}$, \\ Kushal Gohil ${ }^{3}$, Akash Shakya ${ }^{4}$, Mohhamad Umair Attar ${ }^{3}$ \\ ${ }^{1}$ Department of Orthopaedics, Topiwala National Medical College and B.Y.L. Nair Hospital, Mumbai, India \\ ${ }^{2}$ Department of Orthopaedics, Shri Shankaracharya Institute of Medical Science, Bhilai, India \\ ${ }^{3}$ Fortis Hospital, Mohali, India \\ ${ }^{4}$ Department of Orthopaedics, Jagjivanram Railway Hospital, Mumbai, India
}

\section{Study Design: Retrospective study.}

Purpose: To assess the relationship between the severity of lumbar canal stenosis (LCS) and type-Il diabetes mellitus (DM).

Overview of Literature: DM is a multiorgan disorder that has an effect on all types of connective tissues. LCS is a narrowing of the spinal canal with nerve root impingement that causes neurological claudication and radiculopathy. Identification of the risk factors of LCS is key in the prevention of its onset or progression.

Methods: LCS patients were divided into three groups as per DM status: group A without DM ( $n=150)$; group B patients with wellcontrolled DM; and group $C$ patients with uncontrolled DM. Groups $B$ and $C$ were subdivided into group B1: patients with DM with a duration of $\leq 10$ years $(n=76)$, group B2: DM with duration of $>10$ years $(n=68)$, group-C1 DM duration $\leq 10$ years $(n=56)$, and group C2 DM duration $>10$ years $(n=48)$. The severity of LCS was evaluated using the Swiss Spinal Stenosis Scale (SSSS) and Modified Oswestry Disability score (MODS). Operated patients ligamentum flavum sent for histological staining and quantitative immunofluorescence analysis.

Results: The demographic data of groups did not show any difference except in age. There was no difference between the mean SSSS and MODS of groups A and B1. Groups B2, C1, and C2 had higher average SSSS and MODS than group A ( $<<0.05)$. Groups B2 and $\mathrm{C} 2$ had higher SSSS and MODS than groups B1 and C1. Groups $\mathrm{C} 1$ and $\mathrm{C} 2$ had higher scores than groups B1 and B2 $(p<0.05)$. The severity of LCS was significantly related to the duration of DM in groups B and C $(p<0.05)$. Uncontrolled and longer duration of DM had significant elastin fibers loss and also higher rate of disk apoptosis, high matrix aggrecan fragmentation, and high disk glycosaminoglycan content.

Conclusions: Longer duration and uncontrolled diabetes were risk factors for LCS and directly correlate with the severity of LCS.

Keywords: Diabetes mellitus; Lumbar canal stenosis; Swiss Spinal Stenosis Scale; Modified Oswestry Disability score

\section{Introduction}

Diabetes mellitus $(\mathrm{DM})$ is a multiorgan disorder that has an effect on many types of connective tissues including bone and cartilage, and it is characterized by hyperglycemia that has acute as well chronic biochemical and

Received Nov 18, 2020; Revised Jan 19, 2021; Accepted Jan 19, 2021

Corresponding author: Ghanshyam Kakadiya

Department of Spine Surgery, Fortis Hospital, Mohali-160062, India

Tel: +91-7778984811, E-mail: spinenair@gmail.com 
anatomical sequelae [1]. Type-II DM is the most common form of diabetes. According to data from the World Health Organization, the estimated prevalence of DM among the Indian adult population was $11.8 \%$ [2]. Diabetes is associated with an increased risk of certain musculoskeletal pathologies. Prolonged and frequent complications of diabetes include diabetic neuropathy, with symptoms such as pain and sensory and motor deficits in the legs [3]. Lumbar canal stenosis (LCS) is characterized by a narrowing of the spinal canal with nerve root impingement that leads to neurological claudication and radiculopathy. There is hypertrophy of the ligament flavum and facet joints in LCS due to degenerative changes. The pathophysiology of LCS is still to be elucidated. The ligament flavum covers dorsal and lateral parts of the spinal canal and its role is crucial in the pathogenesis of LCS [4]. In diabetic conditions, hypertrophy of the ligamentum flavum is faster and severe [5]. Some studies suggested that there was a significant difference in the incidence and severity of LCS in patients with diabetes compared to those with degenerative disk disease [6]. Therefore, DM was considered as a risk factor for LCS; however, its mechanism is still unclear $[7,8]$. Approximately $13 \%$ of patients who underwent lumbar spine surgery were diagnosed with DM and the prevalence of DM in the same population was $8 \%$ [9]. DM leads to vasculopathy, which might be the effect on the nutrition of the spine and causes disk degeneration and LCS [10]. In patients with $\mathrm{DM}$, one growing problem is the lack of early detection of canal stenosis, which causes many complications. In this retrospective study, we determined the prevalence, and association of DM with LCS with histopathological evidence.

\section{Materials and Methods}

\section{Patients}

After approval from the institutional ethics committee of Topiwala National Medical College and B.Y.L. Nair Hospital, Mumbai, India (ECARP/2016/168), we conducted this retrospective study from 2016 to 2018 at three different tertiary care hospitals in India. We obtained informed consent from all patients prior to participating in the study.

The diagnostic criteria for LCS are the magnetic resonance imaging (MRI) suggested of LCS- narrowing of the canal, lateral recess, and/or foramen. We also confirmed the presence of at least one of these clinical signs of LCS: (1) symptoms worsened by lumbar extension and relieved by flexion, (2) leg pain relived by leaning forward on a shopping cart while walking, and (3) leg symptoms worsened by walking and relieved by sitting.

The inclusion criteria were as follows: (1) age $\geq 40$ and $\leq 65$ years, (2) no history of previous conservative or surgical treatment, (3) LCS on MRI, (4) non-smoker/no tobacco chewing, and (5) no other significant co-morbidity. The exclusion criteria were as follows: (1) extreme spinal loading occupation and (2) history of spinal trauma.

Three hundred and ninety-eight patients were included as per inclusion-exclusion criteria. The patients were divided into three different groups as per DM status. Group A had non-DM patients ( $n=150)$. Group B had patients with controlled DM $(n=144)$ and group C had patients with uncontrolled DM $(n=104)$. Patients with DM were included in groups $\mathrm{B}$ and $\mathrm{C}$ and subdivided as per the duration of DM. Group B1 consisted of patients with controlled DM with a duration of $\leq 10$ years $(n=76)$, group B2 consisted of patients with controlled DM with a duration of $>10$ years $(n=68)$. Group $\mathrm{C} 1$ consisted of patients with uncontrolled DM with a duration of $\leq 10$ years $(n=56)$ and group $\mathrm{C} 2$ consisted of patients with uncontrolled DM with a duration of $>0$ years $(n=48)$. We recorded the demographics, clinical, and radiological data of all patients. The criteria for the diagnosis of DM were fasting plasma glucose $\geq 126 \mathrm{mg} / \mathrm{dL}$, random plasma glucose $\geq 200 \mathrm{mg} / \mathrm{dL}$ and hemoglobin A1c (HbAlc) $\geq 6.5 \%$. We used the Modified Oswestry Disability score (MODS) [11] and Swiss Spinal Stenosis Scale (SSSS) [12] to assess clinical and functional results.

Lumbo-sacral spine antero-posterior and lateral standing radiographs and MRI were performed for LCS diagnosis and scoring. Patients with failed conservative management were managed surgically, and various decompression/spinal stabilizing procedures (laminectomy, transforaminal lumbar interbody fusion) were performed according to the patient's clinical finding. A sample of the disk of patients who were operated on for LCS was sent for histopathological and quantitative immunofluorescence analysis.

\section{Histopathological and immunofluorescence analysis}

We performed (1) histological analysis of the interverte- 


\begin{tabular}{|c|c|c|c|c|c|}
\hline \multirow{2}{*}{ Characteristic } & \multirow{2}{*}{ Group A } & \multicolumn{2}{|c|}{ Group B } & \multicolumn{2}{|c|}{ Group C } \\
\hline & & B1 & B2 & C1 & $\mathrm{C} 2$ \\
\hline No. of patients & 150 & 76 & 68 & 56 & 48 \\
\hline Age (yr) & $54.2 \pm 8.9$ & $57.9 \pm 7.7$ & $60.9 \pm 8.1$ & $58.3 \pm 8.6$ & $61.3 \pm 7.6$ \\
\hline Body mass index $\left(\mathrm{kg} / \mathrm{m}^{2}\right)$ & $19.31 \pm 2.0$ & $19.5 \pm 1.7$ & $18.9 \pm 1.8$ & $19.0 \pm 1.7$ & $20.1 \pm 1.4$ \\
\hline Hemoglobin A1c (\%) & $5.6 \pm 0.2$ & $6.6 \pm 0.4$ & $6.7 \pm 0.4$ & $7.5 \pm 0.6$ & $7.7 \pm 0.5$ \\
\hline Duration of diabetes mellitus (yr) & - & $6.3 \pm 0.7$ & $12.1 \pm 1.2$ & $7.2 \pm 1.1$ & $13.1 \pm 1.5$ \\
\hline \multicolumn{6}{|l|}{ No. of spinal levels involvement } \\
\hline$\leq 2$ & 62 & 50 & 24 & 20 & 22 \\
\hline$\geq 3$ & 88 & 46 & 44 & 36 & 26 \\
\hline
\end{tabular}

Values are presented as number or mean \pm standard deviation.

bral disc sample and ligamentum flavum, (2) proteoglycan synthesis, and (3) apoptosis assay. We collected and analyzed all clinical, functional, biochemical, radiological, and pathological data.

\section{Statistical analysis}

We performed statistical analysis using IBM SPSS ver. 19.0 (IBM Corp., Armonk, NY, USA). All data are presented as mean \pm standard deviation or percentages. Clinical characteristics were compared between the groups using linear model analysis for continuous variables and the chi-square test for categorical data. Multinomial logistic regression analysis was adopted to identify the relationship between DM and the severity of LCS.

\section{Results}

\section{Main results}

Three hundred and ninety-eight patients were included with a mean age of $56.47 \pm 9.71$ years (range, $40-65$ years). There was no significant difference in sex, body mass index and other demographic data among all groups $(p>0.05$, except for age $[p<0.05])$ (Table 1$)$.

The mean SSSS was $54 \pm 10.1$ in group A, 61 \pm 9.12 in group B1, $66 \pm 14.1$ in group B2, $61 \pm 4.10$ in group C1, and $69.6 \pm 9.50$ in group C2. There was no significant difference between the mean SSSS of groups A and B1. Groups B2, C1, and C2 showed higher a SSSS than group A $(p<0.05)$. Uncontrolled DM groups B2 and C2 had a higher SSSS than controlled DM groups. Group C showed
Table 2. Functional and histological data

\begin{tabular}{|c|c|c|c|c|c|}
\hline \multirow{2}{*}{ Variable } & \multirow{2}{*}{ Group A } & \multicolumn{2}{|c|}{ Group B } & \multicolumn{2}{|c|}{ Group C } \\
\hline & & B1 & B2 & $\mathrm{C} 1$ & $\mathrm{C} 2$ \\
\hline No. of patients & 150 & 76 & 68 & 54 & 48 \\
\hline SSSS & $54.0 \pm 10.1$ & $61.0 \pm 9.12$ & $66.0 \pm 14.1$ & $61.0 \pm 4.1$ & $69.6 \pm 9.5$ \\
\hline MODS & $40.6 \pm 19.2$ & $52.6 \pm 10.4$ & $53.5 \pm 15.2$ & $54.0 \pm 12.4$ & $54.0 \pm 12.4$ \\
\hline
\end{tabular}

Values are presented as number or mean \pm standard deviation.

SSSS, Swiss Spinal Stenosis Scale; MODS, Modified Oswestry Disability score.

higher scores than group B $(p<0.05)$. The mean MODS was $40.6 \pm 19.2$ in group A, group B1 had 52.6 \pm 10.4 , group B2 had 53.5 \pm 15.2 , group C1 had $54 \pm 12.4$, and $54 \pm 12.4$ in group C2. Group C showed higher scores than group B $(p<0.05)$. A positive trend was observed between DM duration and severity of LCS by utilizing Spearman correlation analysis (Table 2).

\section{Histopathological and immunofluorescence analysis}

Ninety cross-sections were evaluated for histopathology by confocal microscopy (samples $\times 3$ ).

\section{1) GAG content}

We performed a DMMB assay (dimethylmethylene blue assay) to measure glycosaminoglycan (GAG) content. GAG amount was not significantly different after controlling for age. Findings were qualitatively confirmed by safranin- $\mathrm{O}$ and fast green histopathological staining.

2) Proteoglycan synthesis

It was assessed by measuring 35S-sulfate incorporation 
into a disk and flavum tissue using an organotypic culture system. A significant reduction of OG synthesis was noted in patients with DM.

\section{3) Immunoblot analysis}

The pathologic processes involved in ligament flavum hypertrophy include fibrocartilaginous changes due to the proliferation of type-II collagen, ossification, calcium crystal deposition, degeneration of collagen and elastin fibers, and chondroid metaplasia of fibroblasts.

4) Quantitative microscopic analysis

Quantitative microscopic analysis of ligament flavum and

Table 3. Histological data

\begin{tabular}{|c|c|c|c|c|c|}
\hline \multirow{2}{*}{ Variable } & \multirow{2}{*}{ Group A } & \multicolumn{2}{|c|}{ Group B } & \multicolumn{2}{|c|}{ Group C } \\
\hline & & B1 & B2 & $\mathrm{C} 1$ & $\mathrm{C} 2$ \\
\hline No. of patients & 30 & 15 & 15 & 15 & 15 \\
\hline Elastin fiber loss & $0.69 \pm 0.57$ & $1.50 \pm 0.55$ & $1.80 \pm 0.83$ & $2.10 \pm 0.15$ & $2.40 \pm 0.90$ \\
\hline
\end{tabular}

Values are presented as number or mean \pm standard deviation. elastin fibers showed that ADAMTS4 and ADAMTS5 protein expression were investigated in ligament flavum and disk material section; these were significantly higher in patients with DM and were noted to be highest in longer duration in patients with uncontrolled DM. The mean elastin fiber loss was $0.69 \pm 0.57$ in group $A, 1.5 \pm 0.55$ in group $\mathrm{B} 1,1.8 \pm 0.83$ in group $\mathrm{B} 2,2.1 \pm 0.15$ in group $\mathrm{C} 1$, and highest at $2.4 \pm 0.90$ in group $\mathrm{C} 2$. There was a significantly higher loss in the DM groups than in the non-DM group. Hyperglycemia was positively correlated with elastin fiber loss ( $p=0.048$ ) (Table 3, Fig. 1).

\section{Discussion}

In DM, extracellular matrix changes may differ from the normal aging process in two important ways: increased non-enzymatic cross-link of proteins by sugar glycosylation at lysine residues and decreased rate of proteoglycan synthesis $[8,9]$. The identification of risk factors for LCS is important to prevent or delay its progression. In the present study, we evaluated the association between DM and LCS with histopathological evidence. The study dem-
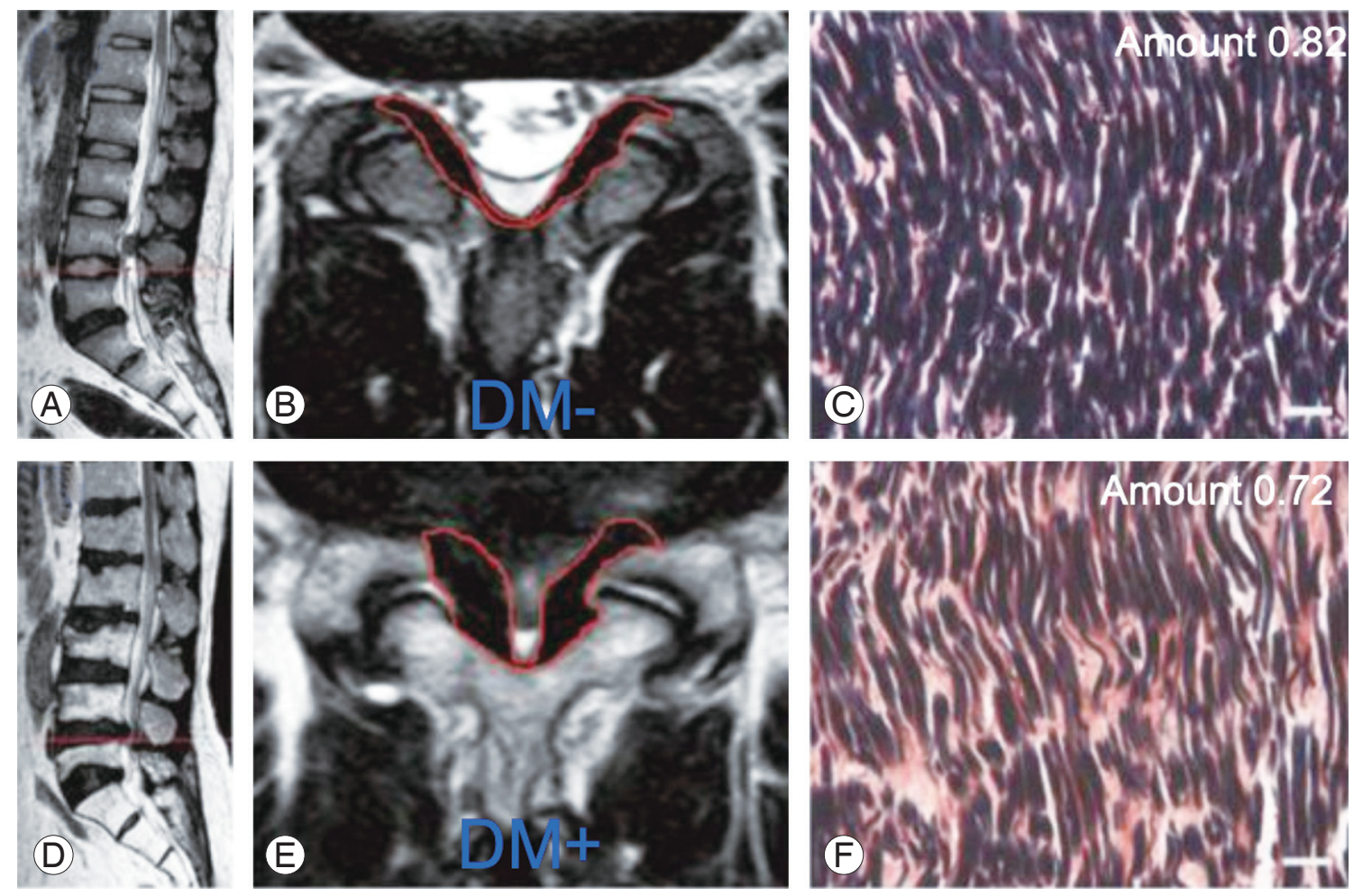

Fig. 1. (A-C) Magnetic resonance imaging and microscopy images of a 58-year-old non-diabetic female showing normal elastin fibers. (D-F) A 60 -year-old female with uncontrolled diabetes mellitus (DM) of longer duration and lumbar canal stenosis shows severe lumbar canal stenosis with moderate loss of elastin fibers. 

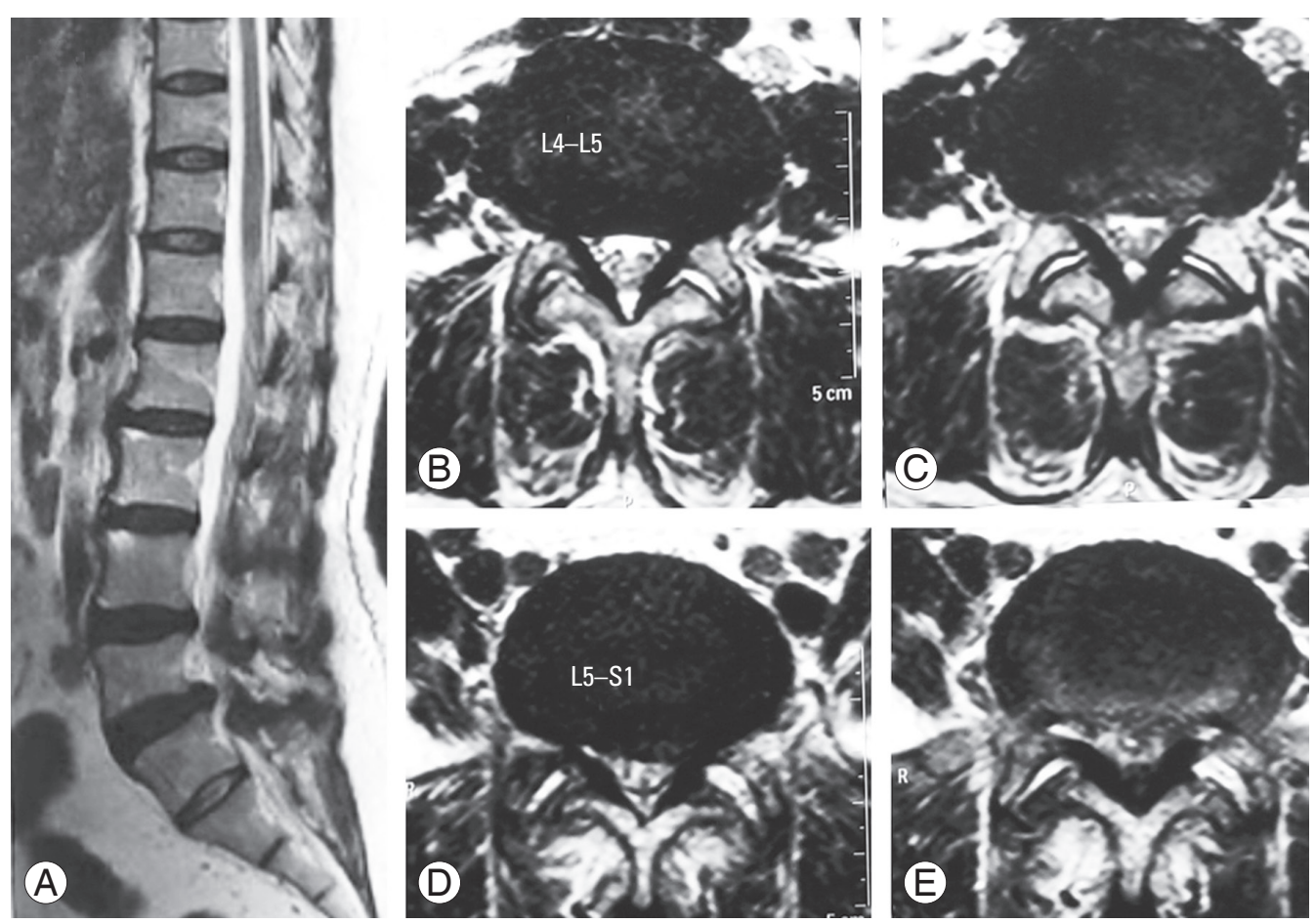

Fig. 2. T2-weighted the lumbar spine magnetic resonance imaging of a 56-year-old controlled diabetic female. (A) Mid-sagittal view shows $L 4-L 5$ disc budge with stenosis. (B-E) Axial scan of L4-L5 and L5-S1 shows disc bulge, ligament flavum hypertrophy, and central with lateral recess stenosis.
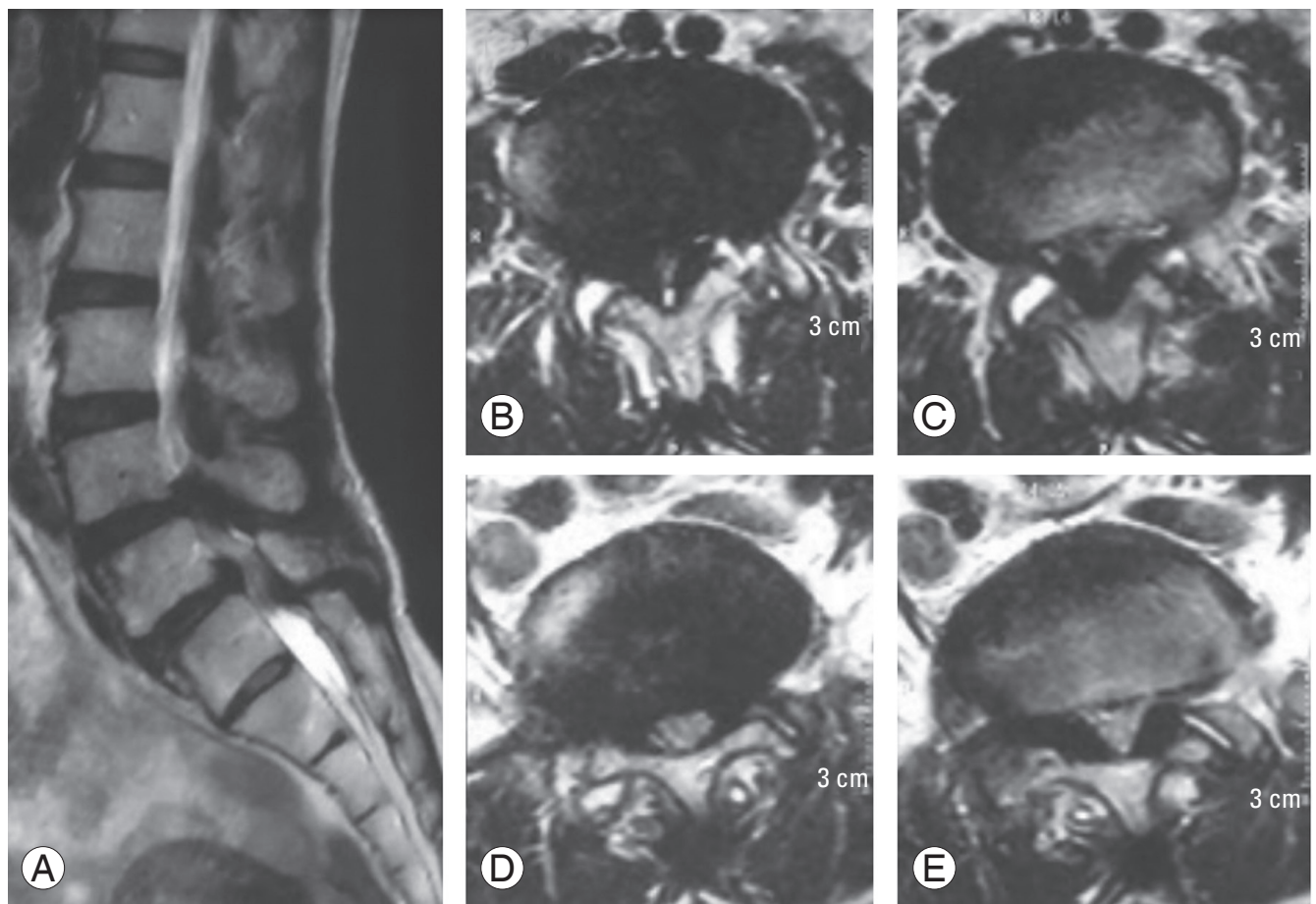

Fig. 3. T2-weighted the lumbar spine magnetic resonance imaging of a 58-year-old uncontrolled diabetic female. (A) Mid-sagittal view shows lumbar degenerative disc with L4-L5and L5-S1 disc budge with stenosis. (B-E) Axial scan shows L4-L5 and L5-S disc bulge, severe ligament flavum hypertrophy, facet joint degeneration-cyst, and severe central with lateral recess stenosis. 
onstrated that patients with DM tended to develop more severe LCS than those without DM. The duration of DM had a positively associated with the severity of LCS, which meant that the longer the duration was, the more severe LCS would be. Uncontrolled DM had more severe LCS. Our results suggest that DM is a risk factor for LCS and such an effect is time and control dependent (Figs. 2, 3).

Frymoyer [13], in his study, concluded that the prevalence of degenerative spondylolisthesis is higher in patients with DM, and some studies found opposite results to this finding [14]. Vogt et al. [14] reported in their study that patients with DM had a poor outcome following lumbar spine surgery than controls, and reoperation rate and prolonged hospitalization were equally significantly high in DM [9]. Some other studies also suggested that high preoperative glycated hemoglobin levels and longer duration of DM were risk factors for poor spinal surgery outcomes $[15,16]$. Several other studies assumed that DM enhances the formation of advanced glycation end products in the nucleus pulposus, which causes progression of disk degeneration $[17,18]$. Chen et al. [10] in their study found that diabetes accelerated the disk degeneration process by microangiopathy. Park et al. [19] and Kong et al. [20] demonstrated in their in vitro studies that autophagy of nucleus pulposus and fibrosis on annulus play important roles in LCS and that they are high in hyperglycemic conditions. Some studies investigated methods of slowing down the process of LCS caused by diabetes. Kong et al. [20] demonstrated that strict blood glucose control is important to prevent and delay the process of LCS. The present study also suggested similar findings; the SSSS and MODS of patients with controlled DM with shorter duration of DM had no significant difference compared to that of non-DM patients.

The microscopic results of the present study also suggest that elastin fiber loss is significant in a longer duration of DM and uncontrolled diabetes. The elastin to collagen fiber ratio decreased with age and results in decreased elasticity and increased stiffness or fibrosis [21]. This loss of elasticity may lead to hypertrophy of the ligament flavum in the lumbar canal and causes compression of the dural sac [11]. Elastin degradation plays a key role in several microvascular and macrovascular complications of diabetes affecting other organs, including retinopathy, nephropathy, and angiopathy $[12,22,23]$. There is also an increase in the expression and activity of various molecules like tissue inhibitors of matrix metalloproteinases, matrix metalloproteinases, platelet derived growth factor $\mathrm{BB}$, connective tissue growth factor, bone morphogenic protein, and inflammatory cytokines [24-26].

Shemesh et al. [27] also concluded similar results for DM and non-DM patients in their study. The exact impact of DM on the pathophysiology of LCS still needs further clarification. The present study is one of the few studies that investigate the relationship between DM and LCS using the functional scoring system with histopathological evidence. HbAlc was used to detect diabetes that increases the accuracy of our study.

Drawbacks of our study are the small date size and short follow-up. Another limitation is the fact that degenerative disk diseases and vertebral osteoporotic fractures may simultaneously exist in the same patient. Prospective comparative studies with large numbers of patients, longer follow-up, and further research of the molecular changes underlying LCS are required for confirmation of our study results.

\section{Conclusions}

Diabetes is positively associated with LCS. DM causes loss of elastin fibers of the ligamentum flavum that leads to fibrosis and hypertrophy. A longer duration of uncontrolled DM is a risk factor for LCS.

\section{Conflict of Interest}

No potential conflict of interest relevant to this article was reported.

\section{Acknowledgments}

The authors would like to acknowledge Professor Dr. Aseem Parekh for giving us opportunity and guidance. We thank the Department of Pathology, TNMC \& BYL Nair Hospital for help.

\section{ORCID}

Ghanshyam Kakadiya: https://orcid.org/0000-0002-03457407; Kalpesh Saindane: https://orcid.org/0000-00022220-6339; Yogesh Soni: https://orcid.org/0000-00025721-5833; Kushal Gohil: https://orcid.org/0000-00016462-3635; Akash Shakya: https://orcid.org/0000-00029752-7209 


\section{References}

1. Kong CG, Park JB, Kim MS, Park EY. High glucose accelerates autophagy in adult rat intervertebral disc cells. Asian Spine J 2014;8:543-8.

2. World Health Organization. Diabetes [Internet]. Geneva: World Health Organization; 2021 [cited 2020 Nov 5]. Available from: https://www.who.int/newsroom/fact-sheets/detail/diabetes.

3. Sakellaridis $\mathrm{N}$. The influence of diabetes mellitus on lumbar intervertebral disk herniation. Surg Neurol 2006;66:152-4.

4. Park JO, Lee BH, Kang YM, et al. Inflammatory cytokines induce fibrosis and ossification of human ligamentum flavum cells. J Spinal Disord Tech 2013;26:E6-12.

5. Robinson D, Mirovsky Y, Halperin N, Evron Z, Nevo $Z$. Changes in proteoglycans of intervertebral disc in diabetic patients: a possible cause of increased back pain. Spine (Phila Pa 1976) 1998;23:849-55.

6. Lotan R, Oron A, Anekstein Y, Shalmon E, Mirovsky Y. Lumbar stenosis and systemic diseases: is there any relevance? J Spinal Disord Tech 2008;21:247-51.

7. Asadian L, Haddadi K, Aarabi M, Zare A. Diabetes mellitus, a new risk factor for lumbar spinal stenosis: a case-control study. Clin Med Insights Endocrinol Diabetes 2016;9:1-5.

8. Anekstein Y, Smorgick Y, Lotan R, et al. Diabetes mellitus as a risk factor for the development of lumbar spinal stenosis. Isr Med Assoc J 2010;12:16-20.

9. Mobbs RJ, Newcombe RL, Chandran KN. Lumbar discectomy and the diabetic patient: incidence and outcome. J Clin Neurosci 2001;8:10-3.

10. Chen S, Liao M, Li J, Peng H, Xiong M. The correlation between microvessel pathological changes of the endplate and degeneration of the intervertebral disc in diabetic rats. Exp Ther Med 2020;20:1.

11. Fairbank JC, Couper J, Davies JB, O'Brien JP. The Oswestry low back pain disability questionnaire. Physiotherapy 1980;66:271-3.

12. Nicoloff G, Baydanoff S, Stanimirova N, Petrova C, Christova P. An association of anti-elastin IgA antibodies with development of retinopathy in diabetic children. Gen Pharmacol 2000;35:83-7.

13. Frymoyer JW. Degenerative spondylolisthesis: diagnosis and treatment. J Am Acad Orthop Surg 1994;2:9-15.
14. Vogt MT, Rubin D, Valentin RS, et al. Lumbar olisthesis and lower back symptoms in elderly white women: the study of osteoporotic fractures. Spine (Phila Pa 1976) 1998;23:2640-7.

15. Sakellaridis N, Androulis A. Influence of diabetes mellitus on cervical intervertebral disc herniation. Clin Neurol Neurosurg 2008;110:810-2.

16. Machino M, Yukawa $Y$, Ito $K$, et al. Risk factors for poor outcome of cervical laminoplasty for cervical spondylotic myelopathy in patients with diabetes. J Bone Joint Surg Am 2014;96:2049-55.

17. Illien-Junger S, Lu Y, Qureshi SA, et al. Chronic ingestion of advanced glycation end products induces degenerative spinal changes and hypertrophy in aging pre-diabetic mice. PLoS One 2015;10:e0116625.

18. Illien-Junger S, Grosjean F, Laudier DM, Vlassara H, Striker GE, Iatridis JC. Combined anti-inflammatory and anti-AGE drug treatments have a protective effect on intervertebral discs in mice with diabetes. PLoS One 2013;8:e64302.

19. Park JS, Park JB, Park IJ, Park EY. Accelerated premature stress-induced senescence of young annulus fibrosus cells of rats by high glucose-induced oxidative stress. Int Orthop 2014;38:1311-20.

20. Kong JG, Park JB, Lee D, Park EY. Effect of high glucose on stress-induced senescence of nucleus pulposus cells of adult rats. Asian Spine J 2015;9:155-61.

21. Zhong ZM, Zha DS, Xiao WD, et al. Hypertrophy of ligamentum flavum in lumbar spine stenosis associated with the increased expression of connective tissue growth factor. J Orthop Res 2011;29:1592-7.

22. Thongboonkerd V, Barati MT, McLeish KR, et al. Alterations in the renal elastin-elastase system in type 1 diabetic nephropathy identified by proteomic analysis. J Am Soc Nephrol 2004;15:650-62.

23. Orasanu G, Plutzky J. The pathologic continuum of diabetic vascular disease. J Am Coll Cardiol 2009;53(5 Suppl):S35-42.

24. Stucki G, Daltroy L, Liang MH, Lipson SJ, Fossel AH, Katz JN. Measurement properties of a self-administered outcome measure in lumbar spinal stenosis. Spine (Phila Pa 1976) 1996;21:796-803.

25. Oh IS, Suh DW, Ha KY. Hypertrophy of the ligament flavum in degenerative lumbar stenosis associated with the increased expression of fractalkine (CX3CL1)/CX3CR1 chemokine. Connect Tissue Res 2013;54:380-5. 
26. Park JB, Chang H, Lee JK. Quantitative analysis of transforming growth factor-beta 1 in ligamentum flavum of lumbar spinal stenosis and disc herniation. Spine (Phila Pa 1976) 2001;26:E492-5.
27. Shemesh S, Sidon E, Kaisler E, et al. Diabetes mellitus is associated with increased elastin fiber loss in ligamentum flavum of patients with lumbar spinal canal stenosis: results of a pilot histological study. Eur Spine J 2018;27:1614-22. 